\title{
Can COVID-19 in pregnancy cause preeclampsia? (Mini commentary on BJOG-20-0800.R1)
}

\author{
Daniel Rolnik ${ }^{1}$ \\ ${ }^{1}$ Monash University
}

June 9,2020

In this issue of BJOG, Mendoza and colleagues report in an observational study the occurrence of a preeclampsia-like syndrome in six out of eight pregnant patients with novel coronavirus disease (COVID-19) who were admitted to the Intensive Care Unit (ICU) with severe pneumonia (Mendoza M, et al. BJOG 2020). There were no symptoms of preeclampsia amongst the 34 pregnant women who had mild forms of COVID-19. Importantly, the authors recorded not only routine laboratory test results, but also measured biophysical and biochemical markers that are typically altered in women with preeclampsia (uterine artery pulsatility index on Doppler ultrasound, serum soluble fms-like tyrosine kinase-1 [sFLT-1] and placental growth factor $[\mathrm{PlGF}])$. Such markers were normal in five of the six cases, in whom the symptoms of preeclampsia resolved after improvement of the maternal clinical situation.

The intriguingly high cumulative incidence of preeclampsia symptoms in women with severe coronavirus disease needs to be interpreted with caution due to the observational nature of the study, the small number of pregnant women with severe infection and the possible role of confounding factors. The normal biomarker results in most cases, nevertheless, suggest that severe coronavirus disease can lead to symptoms that mimic those of preeclampsia in the absence of defective placentation, which is further corroborated by the resolution of the symptoms without the delivery of the placenta when overall clinical improvement occurs. It is plausible that such manifestations are the result of widespread inflammation and endothelial damage, in a process that has been denominated "cytokine storm", responsible for many of the symptoms of the coronavirus-related organ injury (Mehta P, et al. Lancet 2020;395:1033-34) This mechanism includes activation of inflammation pathways that convert arachidonic acid to prostaglandins, thromboxane and eicosanoids, ultimately provoking significant cytokine release. The cascade of events, however, does not appear to influence the levels of specific preeclampsia angiogenic and anti-angiogenic markers such as sFLT-1 and PlGF.

A normal sFLT-1: PlGF ratio in women with clinically suspected preeclampsia can be reliably used predict the short-term absence of disease (Zeisler H, et al. N Engl J Med 2016;374:13-22). Although the definition of preeclampsia has changed over the last 20 years to incorporate less specific clinical features of end-organ damage, biomarkers will likely become part of the disease definition in the years to come or, at least, a valuable tool to select subgroups of women at higher risk of preeclampsia-related morbidity and mortality who require closer monitoring or immediate delivery.

While larger cohorts derived from national datasets or international registries of coronavirus disease in pregnancy will be essential to confirm or refute this association, the preliminary data published in this study indicate that delivery during severe coronavirus disease should not be based on preeclampsia symptoms alone, particularly at early gestational ages, and that the use of ultrasound and serum biomarkers such as the sFLT-1: PIGF ratio might help to guide clinical management by distinguishing hypertension and endothelial dysfunction caused by COVID-19-related inflammation from true preeclampsia.

No disclosures: A completed disclosure of interest form is available to view online as supporting information. 